\title{
ON LOCALLY REPEATED VALUES OF CERTAIN ARITHMETIC FUNCTIONS. III
}

\author{
PAUL ERDÖS, CARL POMERANCE AND ANDRÁS SÁRKÖZY
}

\begin{abstract}
Let $d(n)$ denote the number of natural divisors of $n$. It is shown that the number of $n \leq x$ with $d(n)=d(n+1)$ is $O(x / \sqrt{\log \log x})$. In addition, certain related problems and results are presented.
\end{abstract}

1. Introduction. Let $\nu(n)$ denote the number of distinct prime factors of $n$. Our main result in this paper is that the number of $n \leq x$ with $\nu(n)=\nu(n+1)$ is $O(x / \sqrt{\log \log x})$. We obtain the same result for the equations $\Omega(n)=\Omega(n+1)$ and $d(n)=d(n+1)$, where $\Omega(n)$ is the number of prime factors of $n$ counted with multiplicity and $d(n)$ is the divisor function.

A few years ago, Heath-Brown [7] proved that $d(n)=d(n+1)$ has at least order of magnitude $x /(\log x)^{7}$ solutions $n \leq x$ and that the same is true for $\Omega(n)=\Omega(n+1)$. (It is not clear if the method goes through for $\nu(n)=\nu(n+1)$.) In the second paper in this series [4], we showed that the number of $n \leq x$ for which $|\nu(n)-\nu(n+1)| \leq 3$ is at least of order of magnitude $x / \sqrt{\log \log x}$ and obtained the same result for $\Omega(n)$. For $d(n)$, we have the analogous result for those $n \leq x$ for which $d(n)=2^{i} d(n+1)$ for some integer $i$ with $|i| \leq 3$.

Very recently, Hildebrand [8], combining the methods of [4] and [7], showed that $d(n)=d(n+1)$ has at least order of magnitude $x /(\log \log x)^{3}$ solutions $n \leq x$ and similarly for $\Omega(n)=\Omega(n+1)$. Assuming a quantitative form of the prime $k$-tuples conjecture in Hildebrand's paper gives order of magnitude $x / \sqrt{\log \log x}$ solutions, which would then be best possible, in light of the main results below.

The situation for other common arithmetic functions can be markedly different. In the second paper of this series we showed that the number of $n \leq x$ for which $\phi(n)=\phi(n+1)$ is less than $x / \exp \left((\log x)^{1 / 3}\right)$ for all large $x$, where $\phi$ denotes Euler's function. The same result holds for the sum of the divisors function $\sigma(n)$.

An estimate just a little weaker than our main result can be immediately obtained from a theorem of Barban and Vinogradov (see Elliott [2, Theorem 20.1]) on the joint distribution of $\nu(n)$ and $\nu(n+1)$. Namely, from this theorem, the number of $n \leq x$ with $\nu(n)=\nu(n+1)$ is

$$
O\left(\frac{x \log \log \log x}{\sqrt{\log \log x} \log \log \log \log x}\right) .
$$

We thank R. R. Hall and G. Tenenbaum for this observation.

It seems interesting, though, to remove the $\log \log \log x / \log \log \log \log x$ factor, not only for esthetic reasons, but because $x / \sqrt{\log \log x}$ is almost certainly the correct

Received by the editors March 12, 1986 and, in revised form, July 7, 1986.

1980 Mathematics Subject Classification (1985 Revision). Primary 11N64; Secondary 11A25.

The research by Carl Pomerance was partially supported by an NSF grant.

This paper was written while András Sárközy was visiting the University of Georgia. 
order of magnitude. In fact, in the second paper in this series we conjectured that there is a positive constant $c$ such that the number of $n \leq x$ with $\nu(n)=\nu(n+1)$ is asymptotic to $c x / \sqrt{\log \log x}$.

It might be interesting to see if the methods of this paper can be used to obtain a sharper error term in the Barban-Vinogradov theorem and thus prove an analog of the LeVeque conjecture for joint distributions of additive arithmetic functions (or more modestly, just for $\nu(n), \nu(n+1)$ ).

In the first paper of this series [3] we considered the equation $n+\nu(n)=m+\nu(m)$ for $n \neq m$. The method for treating the equation $\nu(n)=\nu(n+1)$ can be used to get an upper bound for the number of solutions of $n+\nu(n)=m+\nu(m)$. This result and several other problems suggested by our first paper in this series are discussed in $\S 3$.

Finally we wish to mention that P. T. Bateman and C. A. Spiro [1] have given a heuristic argument for the main theorem in this paper.

We shall use the letter $p$ to denote primes. By $P(n)$ and $p(n)$, respectively, we shall mean the largest and smallest prime factor of $n$ for $n>1$; we let $P(1)=$ $p(1)=1$. The constants $c_{1}, c_{2}, \ldots$ are all positive and absolute.

2. The equation $\nu(n)=\nu(n+1)$. In this section we establish our main result.

THEOREM 2.1. The number of $n \leq x$ with $\nu(n)=\nu(n+1)$ is $O(x / \sqrt{\log \log x})$.

ProOF. Let $\mathcal{N}$ denote the set of $n$ with $\nu(n)=\nu(n+1)$ and $x^{1 / 3}<n<x-1$. It suffices to prove the $O$-estimate for $|\mathcal{N}|$. Given $n \in \mathcal{N}$, let us define the integers $a, b, k, l$ by

$$
\begin{aligned}
n+1 & =a k, & P(a) \leq p(k), & a \leq x^{1 / 3}, & a p(k)>x^{1 / 3}, \\
n & =b l, & P(b) \leq p(l), & b \leq x^{1 / 3}, & b p(l)>x^{1 / 3} .
\end{aligned}
$$

We shall assume that $p(k)<p(l)$, the case $p(k)>p(l)$ being similar.

For $1<y \leq x^{1 / 3}$, let $N(y)$ denote the number of $n \in \mathcal{N}$ with $y \leq p(k)<y^{3}$. Thus if $y_{j}=x^{3^{-j}}$, we have

$$
|\mathcal{N}| \leq \sum_{j=1}^{\infty} N\left(y_{j}\right) .
$$

If $j$ is so large that $y_{j}^{3} \leq 2$, then $N\left(y_{j}\right)=0$, so we really only have a finite sum in (2.1).

We now turn our attention to estimating $N(y)$. If $n$ is counted by $N(y)$, then the numbers $a, b, k, l$ defined above satisfy

$$
\begin{gathered}
a k-b l=1, \quad a k<x, \quad \nu(a k)=\nu(b l), \quad P(a) \leq p(k)<y^{3}, \\
y \leq p(k)<p(l), \quad a \leq x^{1 / 3}, \quad b \leq x^{1 / 3}, \quad a>x^{1 / 3} / p(k)>x^{1 / 3} / y^{3} .
\end{gathered}
$$

Since all of the primes in $k, l$ are at least $y$, we have

$$
\nu(k) \leq \frac{\log x}{\log y}, \quad \nu(l) \leq \frac{\log x}{\log y},
$$

so that

$$
|\nu(b)-\nu(a)| \leq \frac{\log x}{\log y}
$$


We now fix the integers $a, b$ appearing in (2.2) and count the number of pairs $k, l$ there can be. Let $k_{0}, l_{0}$ denote the unique integers which satisfy

$$
a k_{0}-b l_{0}=1, \quad 0 \leq k_{0}<b, \quad 0 \leq l_{0}<a .
$$

Thus if $k, l$ satisfy (2.2) we have some integer $m$ with

$$
k=b m+k_{0}, \quad l=a m+l_{0}, \quad 0 \leq m \leq \frac{x}{a b}, \quad p\left(\left(b m+k_{0}\right)\left(a m+l_{0}\right)\right) \geq y .
$$

Thus it suffices to count the number of $m$ satisfying (2.4). This is easily done by using either Brun's method or Selberg's sieve (e.g., see Halberstam and Richert [5, Theorem 3.1, p. 101]; note that $a \leq x^{1 / 3}, b \leq x^{1 / 3}$ by $\left.(2.2)\right)$ which gives that the number of such $m$ is at most

$$
\frac{c_{1} x}{\phi(a) \phi(b) \log ^{2} y}
$$

for some absolute constant $c_{1}$.

Thus from (2.2) and (2.3) we have

$$
\begin{aligned}
N(y) \leq & \sum_{\substack{a>x^{1 / 3} / y^{3} \\
P(a)<y^{3}}} \sum_{\substack{b \leq x^{1 / 3} \\
|\nu(b)-\nu(a)| \leq \log x / \log y}} \frac{c_{1} x}{\phi(a) \phi(b) \log ^{2} y} \\
= & \frac{c_{1} x}{\log ^{2} y} \sum_{\substack{a>x^{1 / 3} / y^{3} \\
P(a)<y^{3}}} \frac{1}{\phi(a)} \sum_{|t-\nu(a)| \leq \log x / \log y} \sum_{\substack{b \leq x^{1 / 3} \\
\nu(b)=t}} \frac{1}{\phi(b)} .
\end{aligned}
$$

The inner sum on $b$ is at most

$$
\begin{aligned}
& \frac{1}{t !}\left(\sum_{p \leq x^{1 / 3}}\left(\frac{1}{\phi(p)}+\frac{1}{\phi\left(p^{2}\right)}+\frac{1}{\phi\left(p^{3}\right)}+\cdots\right)\right)^{t} \\
& \quad=\frac{1}{t !}\left(\sum_{p \leq x^{1 / 3}} \frac{p}{(p-1)^{2}}\right)^{t} \leq \frac{1}{t !}\left(\log \log x+c_{2}\right)^{t} \leq \frac{c_{3} \log x}{\sqrt{\log \log x}}
\end{aligned}
$$

where for the last inequality we used Stirling's formula and then replaced the expression in $t$ with its maximal value. Using this estimate in (2.5), we have

$$
N(y) \leq \frac{c_{4} x \log ^{2} x}{\sqrt{\log \log x} \log ^{3} y} \sum_{\substack{a>x^{1 / 3} / y^{3} \\ P(a)<y^{3}}} \frac{1}{\phi(a)} .
$$

To estimate this sum we prove the following lemma.

LEMMA. There is an absolute constant $c_{5}$ such that if $u>0$ and $v \geq 2$, then we have

$$
\sum_{\substack{n \geq u \\ P(n) \leq v}} \frac{1}{\phi(n)}<c_{5} \log v \exp \left(-\frac{\log u}{2 \log v}\right) .
$$


ProOF. Let $\sigma=1 /(2 \log v)$. Note that if $p \leq v$, then $p^{\sigma} \leq \sqrt{e}$. We have

$$
\begin{aligned}
\sum_{\substack{n \geq u \\
P(n) \leq v}} \frac{1}{\phi(n)} & \leq \frac{1}{u^{\sigma}} \sum_{P(n) \leq v} \frac{n^{\sigma}}{\phi(n)}=\frac{1}{u^{\sigma}} \prod_{p \leq v}\left(1+\frac{p^{\sigma+1}}{(p-1)\left(p-p^{\sigma}\right)}\right) \\
& <u^{-\sigma} \exp \left(\sum_{p \leq v} \frac{p^{\sigma+1}}{(p-1)\left(p-p^{\sigma}\right)}\right)<<u^{-\sigma} \exp \left(\sum_{p \leq v} \frac{p^{\sigma}}{p}\right) \\
& =u^{-\sigma} \exp \left(\sum_{p \leq v} \frac{1}{p}(1+O(\sigma \log p))\right) \\
& =u^{-\sigma} \exp (\log \log v+O(1)) \\
& <<u^{-\sigma} \log v=\log v \exp \left(-\frac{\log u}{2 \log v}\right)
\end{aligned}
$$

which proves the lemma.

Using the lemma in (2.6), we have

$$
N(y) \leq \frac{c_{6} x \log ^{2} x}{\sqrt{\log \log x} \log ^{2} y} \exp \left(-\frac{\log \left(x^{1 / 3} / y^{3}\right)}{6 \log y}\right) .
$$

Recalling that $y_{j}=x^{3^{-j}}$, this gives

$$
N\left(y_{j}\right) \leq \frac{c_{6} x}{\sqrt{\log \log x}} 3^{2 j} \exp \left(-\frac{1}{6}\left(3^{j-1}-3\right)\right) .
$$

Putting this estimate into (2.1) we have our theorem.

REMARKS. The above proof can be made to go through if we replace $\nu(n)=$ $\nu(n+1)$ with $d(n)=d(n+1)$. To do this we first show that the quadruple $a, b, k, l$ appearing in the proof can also be assumed to satisfy

$$
(a, k)=(b, l)=1, \quad k, l \text { square-free. }
$$

Indeed, the $n$ for which these properties fail are negligible. We next replace (2.3) with

$$
\frac{d(a)}{d(b)}=2^{i} \quad \text { for some integer }|i| \leq \frac{\log x}{\log y} .
$$

The inner sum in (2.5) now reads $\sum_{b \leq x^{1 / 3}, d(b)=t}(\phi(b))^{-1}$. To estimate this, let $s(b)$ denote the largest square-full divisor of $b$. Then

$$
\begin{aligned}
\sum_{\substack{b \leq x^{1 / 3} \\
d(b)=t}} \frac{1}{\phi(b)} & =\sum_{\substack{s q u a r e-f u l l \\
d(s) \mid t}} \sum_{\begin{array}{c}
b \leq x^{1 / 3} \\
s(b)=s \\
d(b)=t
\end{array}} \frac{1}{\phi(b)} \\
& \leq \sum_{s \text { square-full }} \frac{1}{\phi(s)} \sum_{\substack{\nu\left(b^{\prime}\right)=\log _{2}(t / d(s)) \\
b^{\prime} \leq x^{1 / 3} / s}} \frac{1}{\phi\left(b^{\prime}\right)} \\
& <<\frac{\log x}{\sqrt{\log \log x}} \sum_{s \text { square-full }} \frac{1}{\phi(s)}<<\frac{\log x}{\sqrt{\log \log x}}
\end{aligned}
$$


The situation for $\Omega(n)=\Omega(n+1)$ can be handled by similar methods. It is slightly easier than the $d(n)=d(n+1)$ case, though, since here it is not necessary to establish (2.7) nor is it necessary to change the form of (2.3).

3. The equation $n+\nu(n)=m+\nu(m)$. In the first paper in this series we gave two proofs that the equation in the title of this section has infinitely many solutions $n \neq m$. The first, relatively simple proof uses the auxiliary function

$$
g(n)=\sum_{\substack{m \leq n \\ m+\nu(m)>n}} 1
$$

the connection being that if $g(n)>g(n+1)$, then there are two integers $m_{1}, m_{2}$ with $m_{1}+\nu\left(m_{1}\right)=m_{2}+\nu\left(m_{2}\right)=n+1$. The other, more complicated proof actually obtains a decent lower bound for the function

$$
F(x)=\#\{(m, n): m<n \leq x, m+\nu(m)=n+\nu(n)\} ;
$$

we show $F(x)>x \cdot \exp (-4000 \log \log x \log \log \log x)$ for all large $x$. We conjectured that $F(x) \sim c_{7} x$ for some positive constant $c_{7}$.

In this section we state some new problems and results concerning the functions $g(n)$ and $F(x)$. First we record the following upper bound for $F(x)$.

THEOREM 3.1. $F(x)=O(x)$.

This theorem can be proved by the general method of Theorem 2.1. The idea is to replace the equation $\nu(n)-\nu(n+1)=0$ with the equation $\nu(n-i)-\nu(n)=i$, where $i$ is an arbitrary positive integer. One then follows the general outline of the proof of Theorem 2.1, but with many new details, to prove that the number of solutions $n \leq x$ corresponding to a given $i<(\log \log x)^{3 / 4}$ is at most

$$
c_{8} \frac{x}{\sqrt{\log \log x}} \exp \left(-\frac{c_{9} i^{2}}{\log \log x}\right),
$$

where $c_{8}, c_{9}$ are positive, absolute constants. For $(\log \log x)^{3 / 4} \leq i \leq 100 \log \log x$, we use the uniform estimate $x \cdot \exp \left(-c_{9} \sqrt{\log \log x}\right)$ and for $i>100 \log \log x$, we use the uniform estimate $O\left(x / \log ^{2} x\right)$. Summing these estimates for $i \leq$ $(1+o(1)) \log x / \log \log x$ then gives $F(x)=O(x)$.

COROLLARY. There is a positive constant $c_{10}$ such that for all large $x$, the number of distinct integers below $x$ of the form $n+\nu(n)$ is at least $c_{10} x$.

We remark that if it could be shown that the set of distinct integers of the form $n+\nu(n)$ has upper density less than 1, a corollary would be that $F(x)>>x$.

Concerning the function $g(n)$ defined above, it is easy to see that the average order of $g(n)$ is $\log \log n+O(1)$. We also have the following result.

THEOREM 3.2. The normal order of $g(n)$ is $\log \log n$.

ProOF. For each $\varepsilon>0$, let

$$
g_{\varepsilon}(n)=\sum_{\substack{m<n-(1+\varepsilon) \log \log n \\ m+\nu(m)>n}} 1 .
$$


Using the Hardy-Ramanujan inequality [6] for the number of $n \leq x$ with $\nu(n)=t$, it is not hard to show that for any $\varepsilon>0$, there is some $\delta>0$ such that

$$
\sum_{n \leq x} g_{\varepsilon}(n)<<x /(\log x)^{\delta} .
$$

Thus the set of $n$ with $g_{\varepsilon}(n)>0$ has density 0 and so $g(n) \leq(1+\varepsilon) \log \log n$ on a set of density 1. From (3.1) and the fact that the average order of $g(n)$ is $\log \log n+O(1)$, it follows that $g(n) \geq(1-\varepsilon) \log \log n$ on a set of density 1 for each fixed $\varepsilon<0$. This proves the theorem.

It seems much harder to say something more precise about the normal behavior of $g(n)$. However, one can prove by the above method that for each $\varepsilon>0$ there is some $K$ such that the lower density of the set of $n$ with

$$
\log \log n-K \sqrt{\log \log n}<g(n)<\log \log n+K \sqrt{\log \log n}
$$

is at least $1-\varepsilon$. The first author believes it likely that $(g(n)-\log \log n) / \sqrt{\log \log n}$ has a normal distribution in asymptotic density. However, the second and third authors believe rather that $g(n)=\log \log n+o(\sqrt{\log \log n})$ on a set of density 1 . To settle this dispute one might estimate the second moment of $g(n)$, but this seems to be not so easy.

The minimal order of $g(n)$ is also an interesting problem. The first author has called $n$ a "barrier" if $m+\nu(m) \leq n$ for all $m<n$. Thus $n$ is a barrier if and only if $g(n)=1$. We conjecture that there are infinitely many barriers, that is, the minimal order of $g(n)$ is 1 . We can prove that the minimal order of $g(n)$ is $O(\log \log \log n)$. This is done largely by arranging via the sieve that the numbers just below $n$ should be free of primes in the interval $\left[(\log \log n)^{2}, \exp \left(\log n /(\log \log n)^{3}\right)\right]$.

Concerning the maximal order of $g(n)$, it is easy to see by the Chinese remainder theorem and by the prime number theorem that

$$
g(n) \geq(1+o(1))\left(\frac{2 \log n}{\log \log n}\right)^{1 / 2}
$$

for infinitely many $n$. This is probably close to being best possible. The completely trivial upper bound is

$$
g(n) \leq(1+o(1)) \frac{\log n}{\log \log n}
$$

for all $n$. This can be rather easily improved to $\left(\frac{1}{2}+o(1)\right) \log n / \log \log n$, but further improvements seem difficult.

Let $h(n)$ denote the number of solutions of $m+\nu(m)=n$. Clearly, $h(n) \leq$ $g(n-1)$, but we cannot prove a better upper bound for $h(n)$ ihan what we get for $g(n)$. Probably $h(n)$ is unbounded, but this too seems difficult. The best we can do in this direction is that $h(n) \geq 2$ infinitely often-this is of course the main result of the first paper in this series.

\section{REFERENCES}

1. P. T. Bateman and C. A. Spiro, private communication.

2. P. D. T. A. Elliot, Probabilistic number theory. Vol. II, Springer-Verlag, New York, 1980.

3. P. Erdös, C. Pomerance and A. Sárközy, On locally repeated values of certain arithmetic functions. I, J. Number Theory 21 (1985), 319-332. 
4. __ On locally repeated values of certain arithmetic functions. II, Acta Math. Hungar. (to appear).

5. H. Halberstam and H. E. Richert, Sieve methods, Academic Press, London, 1974.

6. G. H. Hardy and S. Ramanujan, The normal number of prime factors of a number n, Quart. J. Math. 48 (1917), 76-92.

7. D. R. Heath-Brown, The divisor function at consecutive integers, Mathematika 31 (1984), 141-149.

8. A. Hildebrand, The divisor function at consecutive integers, Pacific J. Math. (to appear).

Mathematical Institute, Hungarian ACademy of Sciences, Budapest, HunGARY (Current address of Paul Erdös and András Sarközy)

Department of Mathematics, University of Georgia, Athens, Georgia 30602 (Current address of Carl Pomerance) 